\title{
Automatic Dynamic Quantification of Oesophagus Position from Intra-cardiac Echocardiography during Atrial Fibrillation Ablation
}

\author{
Federica Lauretti ${ }^{1}$, Rachele Angeletti ${ }^{1}$, Alessandro Del Monte ${ }^{2}$, Corrado Tomasi ${ }^{2}$, Cristiana Corsi ${ }^{1}$ \\ ${ }^{1}$ DEI, Cesena Campus, University of Bologna, Bologna, Italy \\ ${ }^{2}$ Departement of Cardiology, Ospedale Santa Maria delle Croci, Ravenna, Italy
}

\begin{abstract}
Left atrium posterior wall (LAPW) is an essential target for transcatheter radiofrequency ablation (RFA) of atrial fibrillation (AF), but poses problems due to retroatrial structures potentially damaged by RF. Intracardiac echocardiography (ICE) can be integrated with the $3 D$ electro-anatomical map and can give unique real-time anatomical information about all closely-located pericardiac structures. The present study aimed to automatically detect dynamic oesophagus position and its spatial relationship from the LAPW by ICE during RFA. A fast algorithm based on the evaluation of gray-level intensity distribution in the image and was developed to detect distal and proximal oesophagus boundaries. The algorithm was tested in 15 ICE acquisitions. The detected oesophagus boundaries and those manually traced (MT) were compared and in 5 ICE sequences, dynamic tracking of proximal oesophagus boundary was performed. Mean analysis time was 4.5 sec/frame. Detected oesophagus wall positions were in good agreement with MT. Mean minimum dynamic distance between LAPW and oesophagus proximal wall during acquisition was $0.3 \pm 0.2 \mathrm{~mm}$ (range: $0.0-0.6 \mathrm{~mm}$ ). This technique allows automated and accurate dynamic detection of LAPW and oesophagus position in ICE sequences. It represents a first step for dynamic quantification of oesophagus real-time position' changes and its distance from the LAPW to prevent oesophagus injuries.
\end{abstract}

\section{Introduction}

Intracardiac echography (ICE) is used during radiofrequency ablation (RFA) of atrial fibrillation (AF) to reconstruct 3D electro-anatomic maps of left atrium (LA) to monitor potential complications, including oesophageal injuries. In fact, left atrium (LA) posterior wall (LAPW) comprises essential targets for RFA of AF, but poses problems due to a complex anatomy and to retro-atrial structures potentially damaged by RF, mostly the oesophagus and the related plexi and nerves [1]. By the constant direct visualization, potential complications can be monitored. Apart from the dreaded atriooesophageal fistula, which is fortunately rare $(<0.1 \%)$ [2], oesophageal lesions have been described in $1.6-28 \%$ of cases, and endoscopic mucosal lesions are present in up to $20 \%$ of cases $[1,3,4]$. No preventative method has gained wide acceptance yet. Intracardiac echocardiography (ICE) can be integrated with the 3D electro-anatomical maps constructed by CARTO system (Biosense Webster, Diamond Bar, CA, USA), and can give unique real-time anatomical information about all closely-located peri-cardiac structures [5,6].

In clinical practice, however, the positions of LAPW and oesophagus are monitored by manually tracing ICE images which can be transferred onto a 3D electroanatomical map. LAPW and its distance from the oesophagus are only visually assessed. This tracing procedure is imprecise, time-consuming, cumbersome and represents a freezed representation of a dynamic situation. In addition, oesophagus visual assessment does not allow quantitative evaluation of LAPW-oesophagus relationship.

Accordingly, the present study aimed at automatically detect and monitor oesophagus position and its spatial relationship from the LAPW by ICE during RFA of AF.

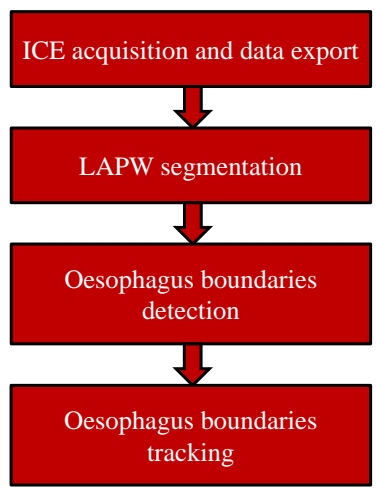

Figure 1. Workflow of the proposed method for oesophagus detection and tracking from ICE sequences. 


\section{Methods}

In this section oesophagus boundaries detection procedure is described (Figure 1).

\subsection{Image acquisition}

Fifteen ICE sequences were acquired in the Electrophysiology Laboratory of Santa Maria delle Croci Hospital in Ravenna, Italy, during AF ablation procedures. Image sequences were stored on an echographic system (ASCUSON Cypress plus, Siemens), then exported in avi format using a magnetic optic device (DynaMO 1300U2, Fujitsu). An algorithm was developed to get each frame from the acquired sequence. Images were acquired when both the atrium and the esophagus were visible in the echo scan.

\subsection{Image processing}

To detect LAPW in each frame we applied a previously developed algorithm aimed at LA segmentation from ICE [7]. Briefly, due to the different amount of noise and artefacts in the LA cavity, acquired sequences were automatically divided into two classes: high noisy images, characterized by high presence of noise inside the LA chamber, and low noisy images, characterized by very low noise inside the chamber. Two different segmentation methods were developed to detect the LAPW in each class of images, the region-based Chan-Vese level-set method (CV) [8] and the clustering K-means algorithm (CL) [9] (Figure 2).
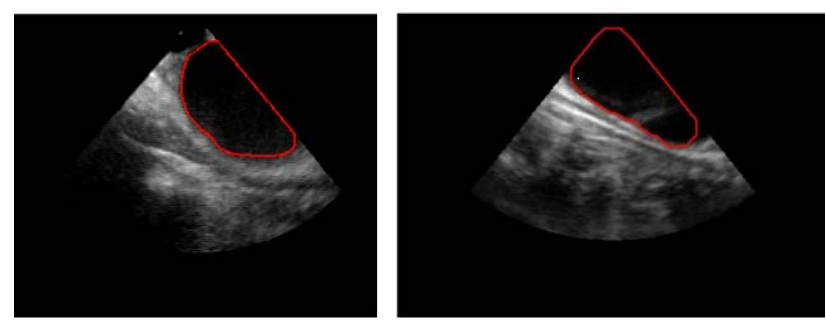

Figure 2. Examples of detected LAPW detection obtained applying the CV model (left panel) and the CL method (right panel).

Once LAPW was detected, a fast algorithm based on the evaluation gray level intensity distribution in the image was developed to detect candidate pixels belonging to the oesophagus proximal wall in the echo scan in which LA mask was subtracted. The research of oesophagus proximal candidate pixels was guided by considering oesophagus anatomy and continuity of his boundaries (Figure 3A). These pixels were the fitted using a forth order model (Figure 3B).

For distal oesophagus boundary detection, considering
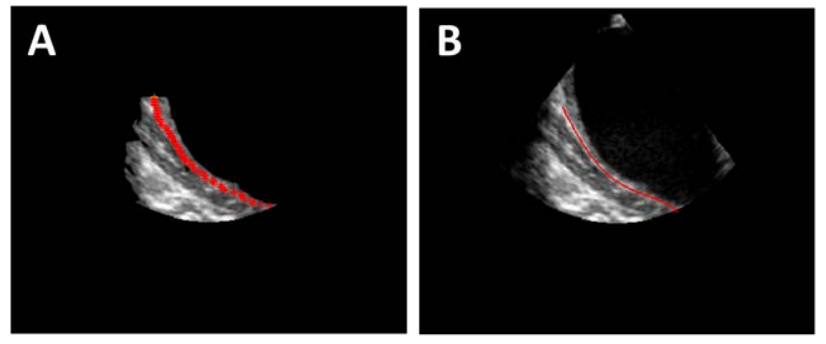

Figure 3. (A) Automated detection of candidate pixels belonging to the oesophagus proximal wall; (B) Result of the forth-order model fitting.
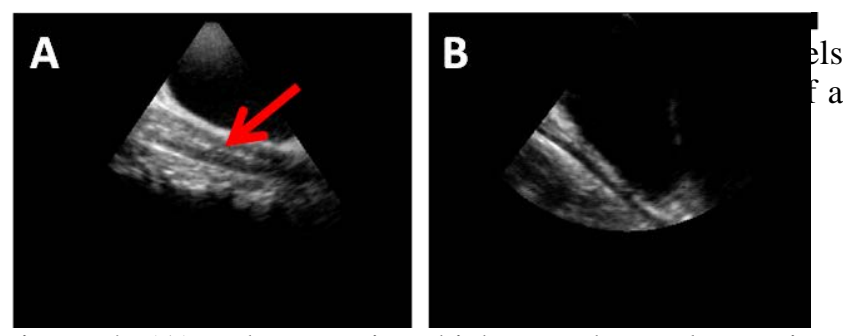

Figure 4. (A) Echo scan in which oesophagus lumen is clearly visible; (B) Echo scan in which oesophagus lumen was not acquired.
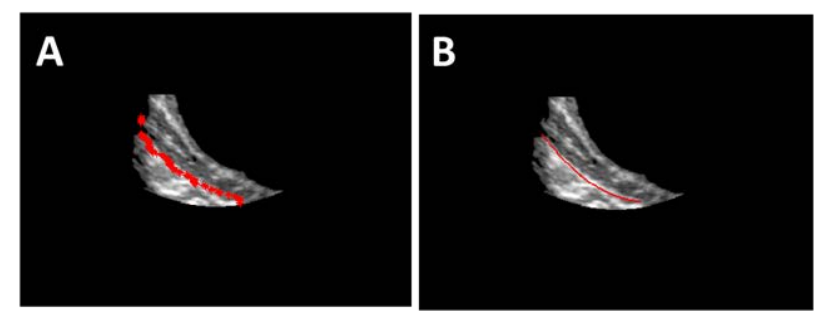

Figure 5. (A) Automated detection of candidate pixels belonging to the oesophagus distal wall; (B) Result of the second-order model fitting.

the variability in ICE scan as well as variability in oesophagus appearance, we first took into account the two cases in which oesophagus lumen could be or not included in the echo scan (Figure 4). A template matching technique was applied to discriminate the two cases for each frame in the acquired ICE sequence. This detection was automatized by applying a template matching algorithm maximizing cross-correlation between image scan line derivatives in the region immediately on the left of the proximal oesophagus boundary. A simple second order model was used for fitting candidate pixels of the distal oesophagus boundary (Figure 5).

Once oesophagus boundaries were correctly detected in the first frame of the ICE sequence, the proximal oesophagus detection procedure was repeated throughout the ICE dynamic acquisition, in each frame in which a cross-correlation measure of similarity between the current frame and the previous one in which oesophagus proximal boundary was detected, was higher than 0.97 . 

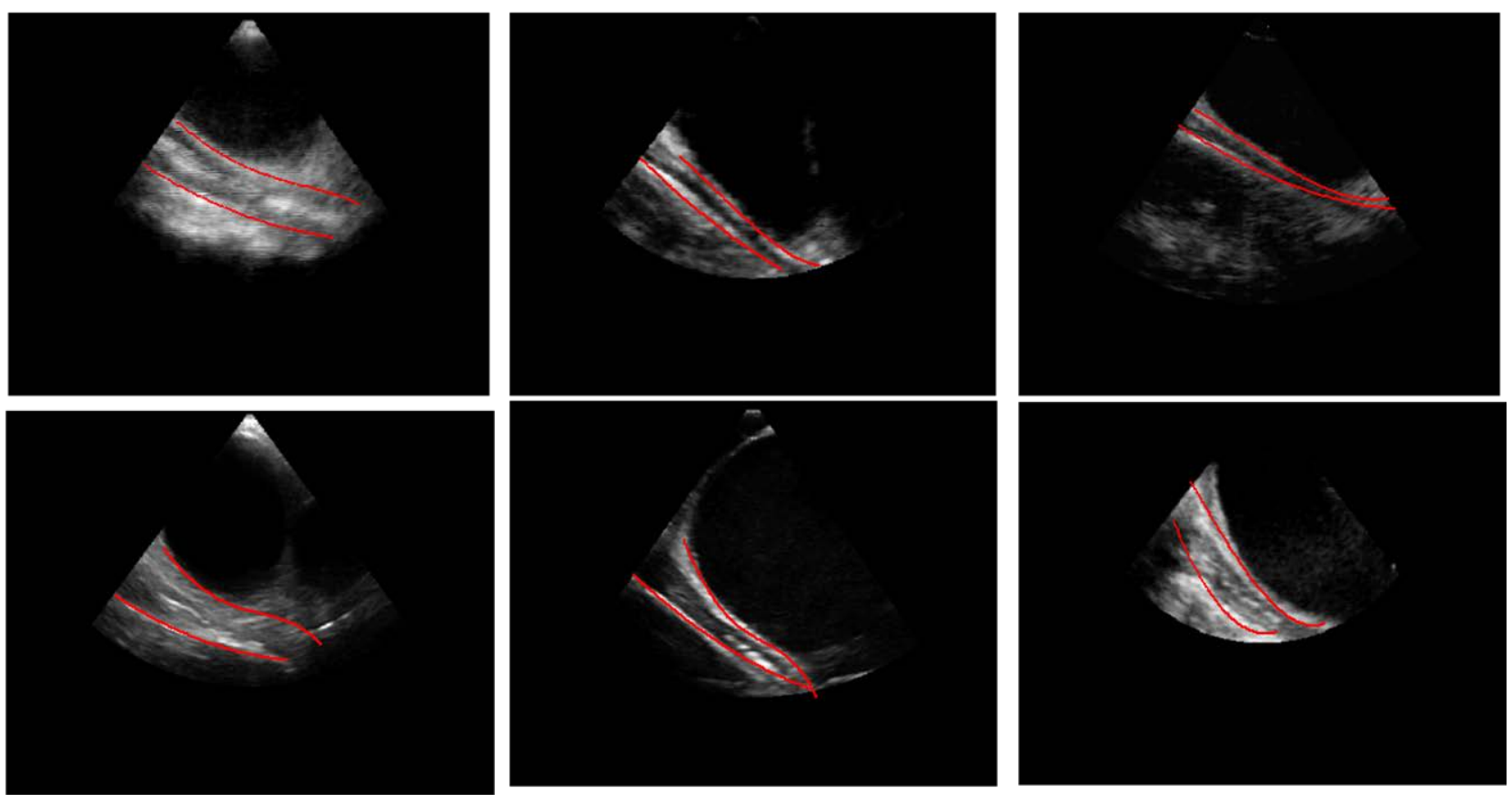

Figure 6. Examples of detected oesophagus boundaries in six different ICE sequences.

This resulted in a dynamic tracking of the oesophagus proximal boundary.

The algorithm was tested on 15 ICE acquisitions. In 15 images, one for each ICE sequence, detected oesophagus boundaries were compared with manually traced (MT) ones by an experienced cardiologist. Mean oesophagus thickness and the distance between esophagus proximal boundary and LAPW was also computed.

In 5 ICE sequences, dynamic tracking of proximal oesophagus boundary was performed and its distance from the LAPW was evaluated throughout the sequence.

\section{Results}

Fifteen ICE sequences were successfully analyzed (10 applying the CV based method and 5 applying the CL based technique) for a total of 3158 frames.

Mean analysis time was $4.5 \mathrm{sec} /$ frame.

Examples of detected oesophagus boundaries in six different ICE sequences are shown in Figure 6.

An example of the comparison between the detected oesophagus contours (in red) and the manually traced oesophagus boundaries by an experienced cardiologist (in green) is shown in Figure 7.

Overall, detected oesophagus positions were in good agreement with MT (bias $=-0.17 \mathrm{~mm}, \mathrm{LOA}: \pm 3,71 \mathrm{~mm}$ ) (see Table 1).

Computed mean average distance between LAPW and oesophagus proximal wall was $2.6 \pm 1.7 \mathrm{~mm}$ (range: 0.6$6.5 \mathrm{~mm}$ ). In this group of fifteen images the mean minimum distance resulted in $0.4 \pm 0.4 \mathrm{~mm}$ (range: $0-1.4$ $\mathrm{mm})$. Mean oesophagus thickness was $6.4 \pm 2.8 \mathrm{~mm}$ (range: 2.8-13.9 mm). Proximal oesophagus wall tracking results in 5 ICE sequences are reported in Table 2.
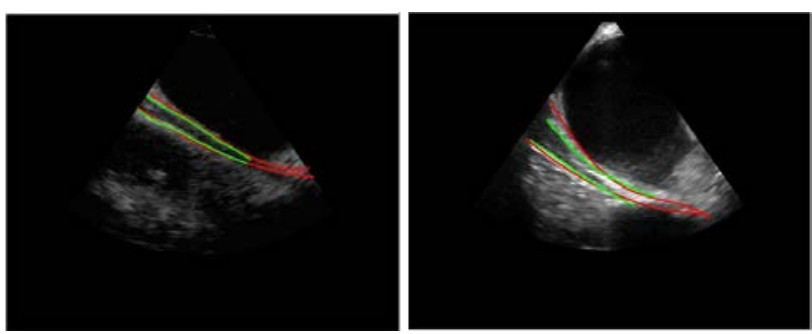

Figure 7. Best (left panel) and worst (right panel) comparison between the detected oesophagus contours (in red) and the manually traced oesophagus boundaries by an experienced cardiologist (in green).

Table 1. Values of the computed similarity indexes between manually traced and automatically detected oesophagus boundaries.

\begin{tabular}{ccc}
\hline & $\begin{array}{c}\text { Proximal oesophagus } \\
\text { boundary }\end{array}$ & $\begin{array}{c}\text { Distal oesophagus } \\
\text { boundary }\end{array}$ \\
\hline Hausdorff & $10,71 \pm 7,11$ & $10,17 \pm 6,59$ \\
distance $[\mathrm{mm}]$ & {$[2,38 \div 26,85]$} & {$[1,64 \div 27,39]$} \\
\hline Minimum & $0,26 \pm 0,63$ & $0,07 \pm 0,1$ \\
distance $(\mathrm{mm})$ & {$[0 \div 2,34]$} & {$[0 \div 0,3]$} \\
\hline Mean & $2,55 \pm 1,98$ & $2,02 \pm 1,43$ \\
distance $(\mathrm{mm})$ & {$[0,51 \div 6,79]$} & {$[0,71 \div 5,98]$} \\
\hline Dice & \multicolumn{3}{c}{$0,71 \pm 0,09$} \\
coefficient & {$[0,55 \div 0,82]$} \\
\hline
\end{tabular}


Table 2. Average values of minimum, maximum and mean LAPW-oesophagus proximal wall distance in 5 ICE sequences.

\begin{tabular}{ccccc}
\hline \multirow{2}{*}{ ICE } & \multirow{2}{*}{$\begin{array}{c}\text { Number of } \\
\text { SEQUENCE }\end{array}$} & \multicolumn{3}{c}{ LAPW-oesophagus proximal wall distance (mm) } \\
\cline { 3 - 5 } & frames & minimum [range] & maximum [range] & mean [range] \\
\hline 1 & 80 & $0,34 \pm 0,34[0,00 \div 1,24]$ & $6,02 \pm 2,18[2,39 \div 10,27]$ & $1,74 \pm 0,30[1,21 \div 2,52]$ \\
\hline 2 & 137 & $0,56 \pm 0,35[0,00 \div 1,18]$ & $5,07 \pm 0,52[4,18 \div 6,7]$ & $1,82 \pm 0,13[1,56 \div 2,15]$ \\
\hline 3 & 71 & $0,57 \pm 0,36[0,00 \div 1,18]$ & $5,04 \pm 0,51[4,18 \div 6,16]$ & $1,82 \pm 0,13[1,61 \div 2,15]$ \\
\hline 4 & 48 & $0,22 \pm 0,25[0,00 \div 1,06]$ & $8,29 \pm 1,86[5,51 \div 13,81]$ & $2,36 \pm 0,51[1,61 \div 4,05]$ \\
\hline 5 & 75 & $0,03 \pm 0,05[0,00 \div 0,29]$ & $4,35 \pm 1,41[1,13 \div 9,48]$ & $1,01 \pm 0,35[0,53 \div 2,31]$ \\
\hline
\end{tabular}

\section{Discussion and conclusion}

Despite the suboptimal quality of the ICE images and the huge variability in echo scan and oesophagus anatomy durin $\mathrm{RFA}$ of $\mathrm{AF}$, the proposed algorithm was able to automatically detect and monitor LAPW and proximal and distal oesophagus boundaries accurately and reliably. It represents a first step for 3D real-time tracking of LAPW and oesophagus placement monitoring during AF RFA to prevent oesophagus injuries.

These preliminary results are promising but further validation on a large number of ICE datasets is necessary, hopefully including data in DICOM format.

\section{References}

[1] Haegeli LM et al., Catheter ablation of atrial fibrillation: an update, Eur Heart J 2014; 35(2):454-9.

[2] Nair GM et al., Atrioesophageal fistula in the era of atrial fibrillation ablation: a review, Can. J Cardiol 2014; 30:388-395.

[3] Martinek $\mathrm{M}$ et al., Esophageal damage during radiofrequency ablation of atrial fibrillation: impact of energy settings, lesion sets, and esophageal visualization, J Cardiovasc Electrophysiol 2009; 20:726-33.
[4] Leite LR et al., Luminal esophageal temperature monitoring with a deflectable esophageal temperature probe and intracardiac echocardiography may reduce esophageal injury during atrial fibrillation ablation procedures: results of a pilot study, Circ Arrhythm Electrophysiol 2011; 4:149-56.

[5] Banchs JE et al., Intracardiac echocardiography in complex cardiac catheter ablation procedures, J Interv Card Electrophysiol, 2010; 28: 167-184.

[6] Filgueiras-Rama D et al., Utility of intracardiac echocardiography for catheter ablation of complex cardiac arrhythmias in a medium-volume training center, Echocardiography,2015;32(4):66070.doi:10.1111/echo.127 14.

[7] Angeletti R, et al. C, Automated Detection of Left Atrium Boundary in Intracardiac Echocardiography During Atrial Fibrillation Ablation, Computing in Cardiology 2015;42:93-96.

[8] Chan T et al., Active Contours Without Edges, IEEE Trans Image Process 2001; 10(2): 266-277.

[9] Hartigan JA et al., A K-Means Clustering Algorithm, Journal of the Royal Statistical Society, Series C, 1979; 28(1):100-108.

Address for correspondence.

Cristiana Corsi, $\mathrm{PhD}$

DEI, University of Bologna

Via Risorgimento 2, 40136 Bologna, Italy

E-mail address: cristiana.corsi3@unibo.it 